\title{
Alcuni risultati di una ricerca sulla densita dei corpi meteorici $(*)$
}

\author{
F. Verniani (**)
}

Ricevuto il 18 Maggio 1963

\begin{abstract}
Riassunto. - Dopo una breve discussione delle cause dell'incertezza attualmente esistente al riguardo della densita e della struttura dei corpi meteorici, viene presentato un metodo per calcolare la densita delle meteore utilizzando dati fotografici. Infine vengono esposti i risultati ottenuti dall'autore analizzando dati pubblicati ad Harvard. Tali risultati mostrano chiaramente che quasi tutte le meteore hanno una densita minore di $1 \mathrm{~g} \mathrm{~cm}^{-3}$ e quindi una struttura porosa in accordo con il modello cometario di Whipple.
\end{abstract}

Summart. - After a brief discussion about the causes of the present uncertainty on the density and structure of meteoroids, a method for computing the meteor density is presented. Eventually the results, obtained from the analysis of photographic data published at Harvard, are shown. Almost all meteors appear to have a density less than $1 \mathrm{~g} \mathrm{~cm}^{-3}$ and a porous structure, in agreement with Whipple's icy-comet model.

\section{I. - INTRODUZIONE.}

Nonostante gli enormi progressi registrati dalla fisica e dall'astronomia delle meteore in questi ultimi anni (***), permane una forte incertezza sulla struttura, sulla composizione e sulla densita delle particelle stesse.

$\left(^{*}\right)$ Nota presentata al $2^{\circ}$ Congresso Internazionale Tecnico Scientifico dello Spazio. Roma, 19-23 Giugno 1962.

$(* *)$ Centro Nazionale per la Fisica dell'Atmosfera del C.N.R. - Roma.

(***) N.d.A. - Per un rapido panorama sull'argomento si veda la relazione presentata dall'autore alla VI Rassegna Elettronica Nucleare del $1959\left({ }^{1}\right)$. 
É noto che fino a pochi anni fa era universalmente ritenuto clie tutte le meteore avessero una composizione chimica e una struttura analoga a quella dei meteoriti; pertanto, essendo la quasi totalita dei meteoriti costituita da silicati, si riteneva che quasi tutte le meteore fossero particelle di natura pietrosa con una densita di circa $3.4 \mathrm{gcm}^{-3}$. Si pensava poi che la parte rimanente, in corrispondenza alla componente ferrosa dei meteoriti, fosse costituita essenzialmente da ferro contenente una piccola percentuale di nichel (densita $\varrho \approx 8 \mathrm{gcm}^{-3}$ ).

Ma nel 1950 Whipple $\left({ }^{2}\right)$ elaboro un modello riguardante la costituzione delle comete che è stato in seguito generalmente accettato: secondo questo modello, il nucleo della cometa deve essere formato da piccole particelle minerali, immerse in un conglomerato di gas $\left(\mathrm{H}_{2} \mathrm{O}, \mathrm{NH}_{3}\right.$, $\mathrm{CHI}_{4}, \mathrm{CO}_{2}, \mathrm{C}_{2} \mathrm{~N}_{2}$ ) allo stato solido. Quando la cometa penetra in una regione dove la radiazione solare comincia ad essere sensibile, le sostanze volatili vaporizzano e liberano le particelle minerali. Le osservazioni spettroscopiche di alcune comete mostrano la presenza di $\mathrm{CN}, \mathrm{C}_{2}, \mathrm{CO}^{+}$ e di altre molecole instabili che possono provenire dalle sostanze stabili solidificate.

Poiche diversi sciami di meteore si possono chiaramente collegare con alcune comete a causa della grande somiglianza dell'orbita, e logico supporre che le particelle costituenti questi sciami provengano dalla disintegrazione delle comete o comunque siano dovute a materiale emesso dalle comete stesse.

Cosi la densita delle meteore di origine cometaria deve essere nettamente minore di $1 \mathrm{gcm}^{-3}$ e la struttura delle stesse deve essere porosa, cioe il corpo meteorico va pensato come qualcosa di spugnoso costituito dagli ordinari materiali contenuti nei meteoriti.

L'ammissione che gran parte dei corpi meteorici abbia una struttura porosa permette anche di spiegare il fenomeno della frammentazione, scoperto da Jacchia nel $1955\left(^{3}\right)$.

Le idee dei maggiori esperti in materia sono pero alquanto discordanti, sia sulla percentuale di meteore porose, sia sulla densita delle stesse. L'incertezza è dovuta anche all'attuale impossibilita di determinare con sicurezza la massa delle meteore. E noto infatti che l'intensita luminosa $I$ emessa da una meteora in una certa regione dello spettro è proporzionale alla energia cinetica perduta dalla meteora nell'unita di tempo:

$$
I=-\frac{1}{2} \tau v^{<} \frac{d m}{d t}
$$


essendo $v$ la velocita della meteora, $m$ la sua massa e $\tau$ l'efficienza luminosa nella regione spettrale considerata. Dalla formula [1] si ha subito il valore $m_{o}$ della massa della meteora fuori dell'atmosfera terrestre:

$$
m_{0}=2 \int_{-\infty}^{+\infty} \frac{i}{\tau v^{2}} d t .
$$

Purtroppo il valore del coefficiente $\tau$ e la sua dipendenza dalla velocita sono noti in modo piuttosto malsicuro; si accetta generalmente per $\tau$ una dipendenza lineare da $v: \tau=\tau_{0} v$ e per $\tau_{0}$ un valore calcolato nel 1933 da Opik (4) in un modo che ha suscitato diverse critiche $\left({ }^{5},{ }^{6}\right)$. Recentemente Cook e Whipple ( $\left.{ }^{7}\right)$ hanno elaborato un nuovo metodo indiretto per determinare le masse delle meteore ma hanno potuto applicarlo in un solo caso. Da quest'unica valutazione essi hanno dedotto che la scala classica delle masse (quella corrispondente al valore di $\tau_{o}$ calcolato da Opik) deve essere moltiplicata per un fattore dell'ordine di 200 e naturalmente per lo stesso fattore deve essere diviso il vecchio valore di $\tau_{o}$.

Ma la difficolta di determinare la densita delle meteore è dovuta anche ad altre cause, come la difficolta di valutare il coefficiente di frenamento e l'energia di ablazione $\xi$ e sopratutto l'impossibilita di conoscere la forma del meteoroide stesso.

Cosi il fatto che le orbite di molte meteore mostrino analogie con quelle delle comete $\left(^{8}\right)$, mentre solo in casi eccezionali si può notare un carattere tipicamente asteroidale - unitamente ai fenomeni di frammentazione mostrati dalle meteore fotografiche meno brillanti - ha portato a ritenere che quasi tutte le meteore abbiano una natura porosa con una densita anche molto bassa e che soltanto poche (alcune delle piu brillanti) siano corpi solidi nel senso ordinario $\left(^{8}\right)$.

La notevole incertezza esistente in questo campo ha spinto l'autore a compiere un lungo studio sulla densita dei corpi meteorici, utilizzando tutti i dati pubblicati. Ne esporremo ora le conclusioni.

\section{II. - EsPoSIZIONE DEI RISULTATI.}

L'autore ha analizzato i dati fotografici riguardanti 512 meteore, pubblicati in massima parte da Whipple $\left({ }^{10}\right)$ e da Hawkins e Southworth $\left({ }^{11}, 12\right)$ : di queste, 137 sono meteore molto brillanti (fireballs) aventi grandezza visuale negativa a le rimanenti 375 sono meteore piil piccole di grandezza positiva. 
I dettagli del procedimento impiegato per il calcolo delle densita, il complesso dei dati riguardanti le meteore brillanti e i risultati ottenuti sono in corso di pubblicazione sul Nuovo Cimento $\left({ }^{13}\right)$; i risultati completi dell'analisi dei corpi piu piccoli saranno anche essi pubblicati tra breve sulla stessa rivista.

Rimandando a tali lavori il lettore che desiderasse maggiori notizie, ci limiteremo qui a ricordare la seguente equazione della teoria dell'ablazione delle meteore $\left({ }^{14}\right)$ :

$$
\frac{Q r_{0}}{\gamma F}=\frac{3\left(v_{0}+12 \xi\right) p_{m} \sec \chi}{8 g \xi}
$$

essendo:

$\varrho=$ densita del meteoroide,

$r_{o}=$ raggio equivalente del meteoroide fuori dell'atmosfera,

$\gamma=$ coefficiente di frenamento medio,

$F$ = fattore di forma (definito da "sezione d'urto del meteoroide" $=F \pi \cdot-$,

$v_{o}$ - velocita della meteora fuori dell'atmosfera,

$\xi=$ energia effettiva di ablazione per unita di massa, definita da $\xi=\gamma l / \Lambda(l=$ calore latente di ablazione per unita di massa; $\Lambda=$ coefficiente di trasferimento del calore),

$p_{m}=$ pressione atmosferica all'altezza $z_{m}$ del massimo di intensita luminosa,

$\chi=$ angolo zenitale della parte osservabile della traiettoria della meteora,

$g=$ accelerazione di gravita.

Le grandezze che compaiono nel secondo membro dell'equazione [3] sono direttamente misurabili come $v_{o}$ e $\chi$ oppure possono essere determinate indirettamente, come $p_{m}$ (da $z_{m}$ e da un modello standard di atmosfera) e come l'energia di ablazione $\xi$.

Così poichè la massa viene determinata mediante l'equazione [2], se si fa l'ipotesi che la media aritmetica dei fattori di forma per un gruppo abbastanza numeroso di meteore sia uguale ad uno, cioe al valore che si avrebbe se tutte le meteore fossero perfettamente sferiche di può determinare la densita delle meteore stesse.

Per l'energia di ablazione $\xi$ sono stati usati i valori forniti da Jacchia (15) e precisamente per la fireballs e stato accettato il valore medio $\xi=16 \mathrm{~km}^{2} \mathrm{~s}^{-2}$ mentre per le meteore piu deboli (grandezza visuale po- 
sitiva) e stata adottata la funzione $\xi=\xi\left(v_{o}\right)$ illustrata in Fig. 1. Molto complicato e stato il calcolo del valore medio del coefficente di frenamento e non è qui il caso di entrare in dettagli.

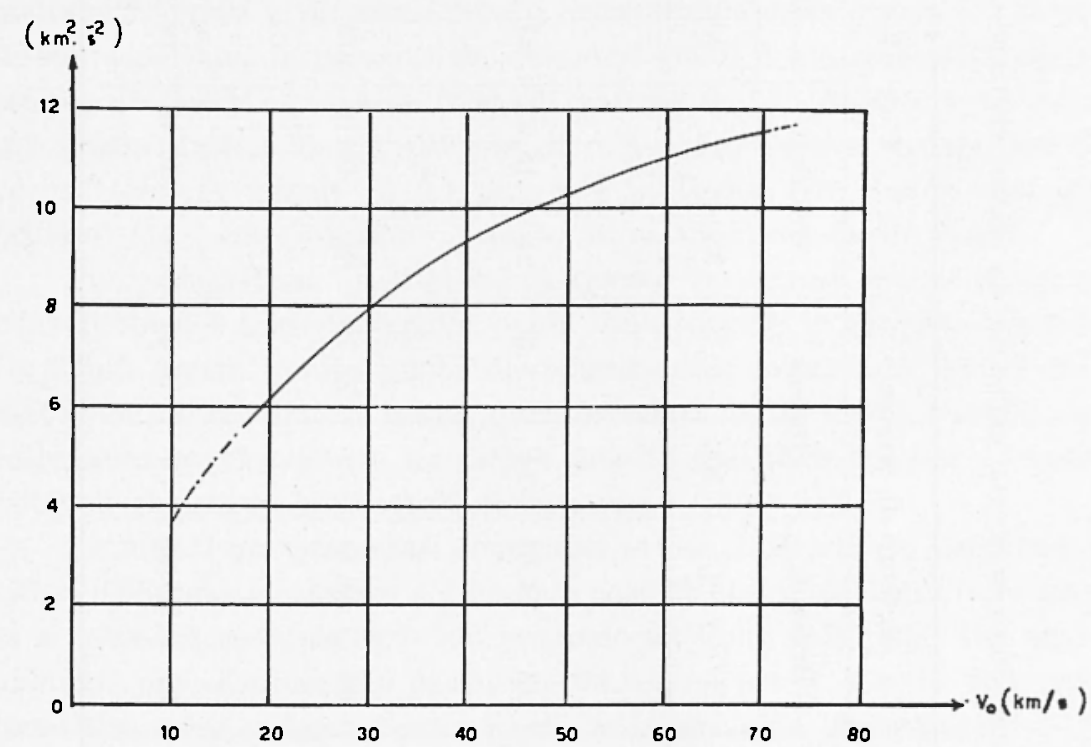

Fig. 1 - Il valore medio di $\xi$ in funzione della velocita $v_{0}$. (Meteore Super-Schimidt; Jacchia 1958)

La Tabella I mostra un quadro riassuntivo globale dei risultati ottenuti sia per le meteore brillanti che per quelle deboli. Le meteore sono state divise in tre grandi classi: corpi compatti, aventi una densita maggiore di $1 \mathrm{gcm}^{-3}$, corpi porosi con una densita compresa tra $0.1 \mathrm{e}$ $0.7 \mathrm{gcm}^{-3} \mathrm{e}$ "dustballs" aventi una densita inferiore a $0.1 \mathrm{gcm}^{-3}$.

Dalla Tabella I risulta chiaramente che la percentuale delle meteore "compatte " e bassa (inferiore al 20\%) mentre la maggior parte delle meteore ha una densita che e in buon accordo col modello della cometa ghiacciata di Whipple, a cui abbiamo accennato.

Occorre dunque abbandonare definitivamente l'idea che le meteore abbiano una struttura fisica analoga a quella dei meteoriti e accettare invece l'idea che prevalentemente esse sono di natura porosa.

I risultati riportati nella Tabella I non mostrano differenze significative nelle percentuali dei vari tipi fra meteore brillanti di sciame e meteore brillanti sporadiche. Si puo notare invece qualche differenza nel caso delle meteore deboli: le meteore aventi un corpo compatto " di tipo classico" sono piu numerose tra le sporadiche che non tra quelle appar- 


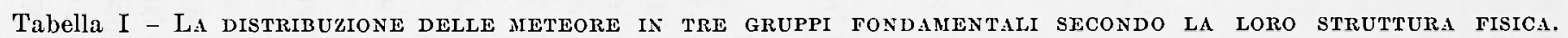

\begin{tabular}{|c|c|c|c|c|c|c|c|c|c|c|c|c|}
\hline & \multicolumn{4}{|c|}{ Meteore sporadiche } & \multicolumn{4}{|c|}{ Meteore di sciame } & \multicolumn{4}{|c|}{ Totale } \\
\hline & \multicolumn{2}{|c|}{ Brillanti } & \multicolumn{2}{|c|}{ Deboli } & \multicolumn{2}{|c|}{ Brillanti } & \multicolumn{2}{|c|}{ Deboli } & \multicolumn{2}{|c|}{ Brillanti } & \multicolumn{2}{|c|}{ Deboli } \\
\hline & No & $\%$ & $\mathrm{No}^{\circ}$ & $\%$ & No & $\%$ & No & $\%$ & No & $\%$ & No & $\%$ \\
\hline $\begin{array}{l}\text { Corpi compatti } \\
\left(\varrho>1 \mathrm{gcm}^{-3}\right)\end{array}$ & 8 & 16.67 & 67 & 23.51 & 13 & 14.61 & 14 & 15.56 & 21 & 15.33 & 81 & 21.60 \\
\hline $\begin{array}{l}\text { Corpi porosi } \\
\left(0.1 \mathrm{gem}^{-3}<\varrho<0.7 \mathrm{gcm}^{-3}\right)\end{array}$ & 22 & 45.83 & 205 & 71.93 & 44 & 49.44 & 70 & 77.78 & 66 & 48.19 & 275 & 73.33 \\
\hline $\begin{array}{l}\text { Corpi estremamente porosi } \\
\quad \text { (dustballs) } \\
\left(\varrho<0.1 \mathrm{gcm}^{-3}\right)\end{array}$ & 18 & 37.50 & 13 & 4.56 & 32 & 35.95 & 6 & 6.66 & 50 & 36.48 & 19 & 5.07 \\
\hline
\end{tabular}


tenenti a qualche sciame, mentre al contrario le porose sono leggermente piu numerose tra le meteore di sciame che non tra le sporadiche.

Piuttosto sorprendente si rivela la notevole differenza esistente tra meteore brillanti e meteore deboli: contrariamente a quanto ci si potrebbe aspettare, la percentuale dei corpi solidi e piu numerosa tra le piccole particelle che danno luogo a meteore deboli che non tra i corpi piu grossi. Inoltre la percentuale di meteore di natura estremamente porosa (densita minore di $0.1 \mathrm{gcm}^{-3}$ ) e piuttosto alta tra le meteore brillanti ( $36 \%$ circa) mentre è molto bassa ( $5 \%$ ) tra quelle deboli.

Una spiegazione qualitativa di questo fatto puo essere ricercata nella probabile frammentazione a cui sono soggetti in maggior misura $i$ corpi piu grossi: questo punto di fondamentale importanza andra studiato con molta cura; un esame della correlazione tra massa e grandezza fotografica (o visuale) da un lato e densita dall'altro dovrebbe fornire notevoli elementi charificatori al riguardo.

I risultati qui presentati sono stati ottenuti prendendo per buoni i valori delle masse calcolati col vecchio coefficiente di efficienza luminosa; se si introducesse il fattore 200 proposto da Cook e Whipple del quale abbiamo precedentemente parlato, otterremmo per la densita delle meteore dei valori estremamente bassi, assolutamente inaccettabili.

Si puo quindi affermare che il valore di $\tau_{0}$ calcolato da Opik da il giusto ordine di grandezza, anche se certamente la sua precisione e scarsa. Una tale conclusione e avvalorata da un lavoro di Mc Crosky ( $\left.{ }^{16}\right)$, che dall'analisi dei dati riguardanti una meteora sicuramente asteroidale ha trovato per $\tau_{o}$ un valore compatibile con quello di Opik.

La determinazione dell'efficienza luminosa con i metodi moderni della fisica quantistica appare estremamente difficile, dato il gran numero di casi in cui può essere emessa luce nell'interazione di un atomo evaporato dalla meteora con una molecola d'aria.

Un po' meno complicato si presenta invece il problema di determinare teoricamente la probabilità di ionizzazione $\hat{\sigma}$ che un atomo, distaccatosi dal corpo meteorico, produca un elettrone libero: da questa, attraverso una relazione empirica che lega $\tau$ con $\beta$ potra essere trovato un valore di $\tau_{0}$ piu attendibile e la giusta dipendenza di $\tau$ dalla velocita.

E opportuno concludere questa breve relazione ricordando la necessità di svolgere un calcolo analogo per le meteore piu piccole, osservabili solamente mediante un radar. I risultati che scaturiranno dallo studio della densita delle radiometeore dovrebbero consentire di determinare, se essa esiste, la massa minima (e quindi la dimensione minima) sotto la quale le particelle meteoriche tornano ad avere la normale consistenza. 


\section{BIBLIOGRAFIA}

(1) Verniani F., Atti uff. del Congresso Scientifico Sezione Elettronica della VI Rassegna Internaz. Elettronica e Nucleare, (1959).

(2) Whipple F. L., Astrophys. J., 111, 375 (1950).

(3) J ACchin I. G., Astrophys. J. 121, 521 (1955).

(4) UPIK E. J., Harvard Reprint Series I No. 100 (1933).

(5) Thomas R. N. and Whipple F. L., Astrophys. J., 114, 448 (1951).

$\left.{ }^{8}\right)$ Whipple F. L. and Hawkins G. S., Handbuch der Physik 52; Astrophysik 3 (1959).

(7) Whipple F. L., Harvard Reprint No. 465 (1957).

(8) JACchia L. G. and Whipple F. L., Smithsonian Contributions to Astrophysics 4, 97 (1961).

$\left({ }^{9}\right)$ Mc Kinfey D.W.R., "Neteor Science and Engineering”, (New York 1961).

(10) Whipple F. L., Astron. J., 59, 201 (1954).

(11) Hawkins G. S. and Soutiworti R. B., Smithsonian Contributions to Astrophysics, 2, 349 (1958).

(12) Hawkins G.S. and Southwortil R. B., Smithsonian Contributions to Astroplysics 4, 85 (1961).

(13) Verniani F., Nuovo Cimento, 26, 209, (1962).

(14) Verniani F., Nuono Cimento, 19, 415 (1961).

(15) JaCchia L. G., Smithsonian Contributions to Astrophisics 2, 181 (1958).

(16) Ic Crosky R. E., Astron. J., 65, 493 (1960). 